\title{
Mechanistic Basis for the Differential Effects of Rivaroxaban and Apixaban on Global Tests of Coagulation
}

\author{
Paul Y. Kim ${ }^{1,2, *}$ Calvin H. Yeh ${ }^{1,2,3, *}$ Brian J. Dale ${ }^{2,4}$ Beverly A. Leslie ${ }^{1,2}$ Alan R. Stafford ${ }^{1,2}$ \\ James C. Fredenburgh ${ }^{1,2}$ Jack Hirsh ${ }^{1}$ Jeffrey I. Weitz ${ }^{1,2,3}$
}

${ }^{1}$ Department of Medicine, McMaster University, Hamilton, Ontario, Canada

2 Thrombosis and Atherosclerosis Research Institute, Hamilton, Ontario, Canada

${ }^{3}$ Department of Biochemistry and Biomedical Sciences, McMaster University, Hamilton, Ontario, Canada

${ }^{4}$ School of Pharmacy and Medical Sciences, University of

South Australia, Adelaide, Australia

TH Open 2018;2:e190-e201.

\begin{abstract}
Address for correspondence Jeffrey I. Weitz, MD, Thrombosis and Atherosclerosis Research Institute, 237 Barton Street E, Hamilton, Ontario, Canada L8L 2X2 (e-mail: weitzj@taari.ca).
\end{abstract}

\begin{abstract}
Keywords

- DOAC

- inhibition kinetics

- coagulation assays

- coagulation inhibitors

Rivaroxaban and apixaban are both small molecules that reversibly inhibit factor Xa. Compared with rivaroxaban, apixaban has minimal effects on the prothrombin time and activated partial thromboplastin time. To investigate this phenomenon, we used a factor Xa-directed substrate in a buffer system. Although rivaroxaban and apixaban inhibited factor $\mathrm{Xa}$ with similar $\mathrm{K}_{\mathrm{i}}$ values at equilibrium, kinetic measurements revealed that rivaroxaban inhibited factor Xa up to 4 -fold faster than apixaban $(p<0.001)$. Using a discontinuous chromogenic assay to monitor thrombin production by prothrombinase in a purified system, rivaroxaban was 4 -fold more potent than apixaban ( $K_{\mathrm{i}}$ values of $0.7 \pm 0.3$ and $2.9 \pm 0.5 \mathrm{nM}$, respectively; $p=0.02$ ). Likewise, in thrombin generation assays in plasma, rivaroxaban prolonged the lag time and suppressed endogenous thrombin potential to a greater extent than apixaban. To characterize how the two inhibitors differ in recognizing factor $\mathrm{Xa}$, inhibition of prothrombinase was monitored in real-time using a fluorescent probe for thrombin. The data were fit using a mixed-inhibition model and the individual association and dissociation rate constants were determined. The association rates for the binding of rivaroxaban to either free factor Xa or factor Xa incorporated into the prothrombinase complex were 10- and 1,193-fold faster than those for apixaban, respectively, whereas dissociation rates were about 3 -fold faster. Collectively, these findings suggest that rivaroxaban and apixaban differ in their capacity to inhibit factor $\mathrm{Xa}$ and provide a plausible explanation for the observation that rivaroxaban has a greater effect on global tests of coagulation than apixaban.
\end{abstract}

\section{Introduction}

Rivaroxaban and apixaban are oral factor Xa inhibitors that were developed as alternatives to warfarin for the prevention

\footnotetext{
These authors contributed equally.
}

and treatment of arterial and venous thrombosis. ${ }^{1}$ These direct, non-vitamin $\mathrm{K}$ antagonist oral anticoagulants (DOACs) are licensed in the United States and Europe for stroke prevention in patients with atrial fibrillation ${ }^{2,3}$ and for the treatment of venous thromboembolism, ${ }^{1,4-6}$ and have experienced rapid adoption in clinical practice. ${ }^{7,8}$ received

January 16, 2018

accepted after revision

March 26, 2018
DOI https://doi.org/

$10.1055 / \mathrm{s}-0038-1649507$. ISSN 2512-9465. (c) 2018 Georg Thieme Verlag KG
Stuttgart · New York

License terms

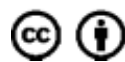


The oral factor Xa inhibitors exhibit a similar mechanism of action. As small molecules that bind reversibly to the active site of factor Xa, rivaroxaban, and apixaban inhibit the enzyme with high affinity as evidenced by their sub-nanomolar inhibition constant $\left(\mathrm{K}_{\mathrm{i}}\right)$ values. ${ }^{9,10}$ In addition to inhibiting free factor $\mathrm{Xa}$, these agents inhibit factor Xa incorporated within prothrombinase, the complex of factor $\mathrm{Xa}$ and factor Va that assembles on activated platelets and converts prothrombin to thrombin. ${ }^{9,10}$ Prothrombinase is the central effector of clotting because its assembly induces structural changes in factor Xa that increase the catalytic efficiency of prothrombin activation by over 100,000 -fold. ${ }^{11}$ Prothrombinase propagates coagulation by rapidly generating thrombin at sites of vascular injury. Therefore, the anticoagulant activity of rivaroxaban and apixaban reflects their rapid association with factor $\mathrm{Xa}$ incorporated within the prothrombinase complex.

Despite their similar affinities for factor Xa, rivaroxaban prolongs the prothrombin time (PT) and activated partial thromboplastin time (aPTT) more than apixaban. ${ }^{12-18}$ A recent study by Jourdi et al reported that rivaroxaban binds free factor Xa with a 4-fold higher on-rate than apixaban and a 1.5-fold lower $\mathrm{K}_{\mathrm{d}}$, and modeling data suggested that this phenomenon explains the greater effect of rivaroxaban on the PT. ${ }^{19}$ This difference is thought to contribute to the superiority of rivaroxaban in the thrombin generation assay. ${ }^{19,20}$ However, the DOACs may have different inhibitory effects on factor Xa when it is incorporated into the prothrombinase complex and its substrate is prothrombin rather than a low-molecular-weight substrate. ${ }^{9,10}$ Therefore, we compared rivaroxaban and apixaban in terms of their affinities for free factor Xa and for factor Xa incorporated into the prothrombinase complex and their rates of inhibition of prothrombinase-induced thrombin generation and we related these effects to those on the PT, aPTT, and thrombin generation assay.

\section{Materials and Methods}

\section{Materials}

Human prothrombin, factor Va, and dansylarginine-N-(3ethyl-1,5-pentanediyl)amide (DAPA) were purchased from Haematologic Technologies (Essex Junction, Vermont, United States), whereas factor Xa and thrombin were purchased from Enzyme Research Laboratories (South Bend, IN). A variant prothrombin molecule cleaved solely at Arg320 (R155A, R271A, R284A, rMZ) was used to generate a stable recombinant form of meizothrombin, which was expressed in BHK cells and isolated as described previously. ${ }^{21}$ The factor Xa-directed chromogenic substrate, Z-D-Arg-Gly-Arg- $p$ nitroaniline (S-2765), was purchased from Chromogenix (Milano, Italy), whereas the thrombin-directed substrate, Tos-Gly-Pro-Arg-pNA (Chromozym-thrombin [Chz-Th]), was from Hyphen BioMed (Neuville-sur-Oise, France). Fluorogenic factor Xa substrate Pefafluor Xa was from Pentapharm (Basel, Switzerland). Z-Gly-Gly-Arg-7-amino-4methylcoumarin (GGR-AMC) and hirudin were from Bachem Bioscience, Inc. (Philadelphia, Pennsylvania, United States). Phospholipid vesicles were prepared in a $3: 1$ ratio of phos- phatidylcholine and phosphatidylserine (PCPS) and stored in $10 \%$ sucrose at $-80^{\circ} \mathrm{C}$ as described previously. ${ }^{22,23}$ RecombiPlasTin $2 \mathrm{G}$, which contains recombinant tissue factor at a concentration of $0.3 \mu \mathrm{g} / \mathrm{mL},{ }^{24}$ was from Instrumentation Laboratory (Bedford, Massachusetts, United States). Prionex was from Pentapharm (Basel, Switzerland).

Rivaroxaban and apixaban, obtained from Suzhou Howsine Biological Technology Company (Suzhou, China), exhibited single and distinct peaks by HPLC analysis. After dissolving the agents in 100\% dimethyl sulfoxide (DMSO) to a concentration of $10 \mathrm{mg} / \mathrm{mL}$, they were stored in aliquots at $-80^{\circ} \mathrm{C}$. Molar concentrations were calculated using molecular weights of 435.9 and 459.5 for rivaroxaban and apixaban, respectively. Concentrations of rivaroxaban and apixaban were confirmed with the Rotochrom chromogenic anti-Xa assay (Diagnostica Stago, Parsippany, New Jersey, United States), which was performed using a ST4 coagulometer (Diagnostica Stago) with commercial rivaroxaban or apixaban calibrators (Technoclone, Vienna, Austria).

To prepare pooled normal human plasma (PNP), $50 \mathrm{~mL}$ of blood was collected from the antecubital veins of 10 to 15 healthy volunteers into a syringe containing $0.105 \mathrm{M}$ sodium citrate. Platelet-poor plasma was obtained as described previously, with the exception that $2,500 \times g$ was used for centrifugation. $^{24}$

\section{Prothrombin Time and Activated Partial Thromboplastin Time}

Stock solutions of rivaroxaban and apixaban were diluted in PNP to $500 \mathrm{ng} / \mathrm{mL}$ ( 1,150 and $1,068 \mu \mathrm{M}$, respectively) and then serially diluted with PNP to a final concentration of $31.25 \mathrm{ng} / \mathrm{mL}$ (72 and $68 \mathrm{nM}$, respectively). The ranges of inhibitor concentrations were chosen to encompass the peak and trough plasma levels measured in patients treated with these agents. ${ }^{25,26}$ The PT was performed using HemosIL HS PLUS (Instrumentation Laboratory, Lexington, Massachusetts, United States) on an ACL 7000 coagulometer (Instrumentation Laboratory) according to the manufacturer's instructions. The aPTT was performed using Actin FSL reagent (Siemens, Marburg, Germany) on a BCS XP analyzer (Siemens) according to the manufacturer's instructions. Assays were performed three times in duplicate at $37^{\circ} \mathrm{C}$. These PT and aPTT reagents were chosen because of their wide availability and their reported sensitivities to rivaroxaban and apixaban. ${ }^{15,27}$

\section{Plasma-Based Thrombin Generation Assays}

Thrombin generation assays were performed in 96-well microtiter plates at $37^{\circ} \mathrm{C}$ as previously described ${ }^{22,24,28}$ using final concentrations of $3 \mathrm{pM}$ tissue factor (HemosIL HS PLUS), $4 \mu \mathrm{M}$ PCPS, $0.5 \mathrm{mM}$ Z-Gly-Gly-Arg-AMC, and $7.5 \mathrm{mM} \mathrm{CaCl}_{2}$, without added corn trypsin inhibitor. Studies were done in the absence or presence of rivaroxaban or apixaban at concentrations up to 2,300 and $2,120 \mu \mathrm{M}(1,000 \mathrm{ng} / \mathrm{mL})$, respectively. Assays were calibrated using Technothrombin TGA thrombin calibrators (Technoclone) according to the manufacturer's instructions with high and low thrombin concentration controls included in each run. The reactions were monitored over time using SpectraMax M5 fluorescence plate reader 
(Molecular Devices, Sunnyvale, California, United States) at excitation and emission at 360 and $460 \mathrm{~nm}$, respectively, with the cutoff filter at $455 \mathrm{~nm}$. All samples and controls were tested in quadruplicate.

\section{Determination of Inhibition Constants of Prothrombinase-Induced Thrombin Generation}

To determine their inhibitory effects on prothrombinaseinduced thrombin generation, rivaroxaban or apixaban was preincubated with prothrombin at $25^{\circ} \mathrm{C}$ prior to addition to a solution containing factor Xa, factor Va, PCPS, and calcium. Reactions were performed in clear or black flat-bottom 96well polystyrene plates (Corning Inc., Corning, New York, United States). The final activation solution consisted of $20 \mu \mathrm{L}$ of 0 to $200 \mathrm{nM}$ inhibitor, $0.5 \mathrm{nM}$ factor Xa, $5.5 \mathrm{nM}$ factor Va, $50 \mu \mathrm{M}$ PCPS, $500 \mathrm{nM}$ prothrombin, and $5 \mathrm{mM}$ $\mathrm{CaCl}_{2}$ in $20 \mathrm{mM}$ HEPES, $150 \mathrm{mM} \mathrm{NaCl}$, pH 7.4 (HBS) containing $0.01 \%$ Tween-80 (HBST). Reactions were stopped after 20 seconds by addition of $20 \mu \mathrm{L}$ of $10 \mathrm{mM}$ EDTA, and thrombin activity was measured by adding $60 \mu \mathrm{L}$ of $500 \mu \mathrm{M}$ Chz-Th and monitoring its hydrolysis at $405 \mathrm{~nm}$ using a plate reader. The rates of Chz-Th hydrolysis were converted to concentrations of thrombin generated using a specific activity of 17.2 $\mathrm{mOD} \cdot \mathrm{min}^{-1} \cdot \mathrm{nM}^{-1}$, which was determined in a separate experiment. Thrombin concentrations were divided by reaction time to obtain the rate of thrombin formation $(\mathrm{nM} / \mathrm{s})$ and divided by the factor Xa concentration to obtain the turnover number $\left(\mathrm{k}_{\mathrm{cat}}\right)$. The turnover number was then plotted against the concentration of inhibitor, and the data were fit to a rectangular hyperbola equation by nonlinear regression analysis using TableCurve version 4.04 (Jandel Scientific):

$$
\mathrm{V}=\mathrm{V}_{0}+\left(\mathrm{V}_{\min }[\mathrm{I}]\right) /\left(\mathrm{IC}_{50}+[\mathrm{I}]\right)
$$

where $\mathrm{V}_{0}$ and $\mathrm{V}_{\text {min }}$ are the turnover numbers of thrombin generation in the absence and presence of saturating concentrations of inhibitor, respectively; $\mathrm{IC}_{50}$ is the concentration of inhibitor required to produce half-maximal reduction; and [I] is the inhibitor concentration. $\mathrm{K}_{\mathrm{i}}$ values were then obtained from the $\mathrm{IC}_{50}$ value using the ChengPrusoff equation:

$$
\mathrm{IC}_{50}=\left[1+\left([\mathrm{S}] / \mathrm{K}_{\mathrm{M}}\right)\right] \mathrm{K}_{\mathrm{i}}
$$

where [S] is the prothrombin concentration and $\mathrm{K}_{\mathrm{M}}$ is the Michaelis constant of prothrombinase for prothrombin (157.4 $\mathrm{nM}$ ) determined in a separate experiment as described previously. ${ }^{21}$

\section{Determination of Steady-State Inhibition Constants of Factor Xa}

To measure the affinity of the inhibitors for factor Xa at equilibrium, $10 \mu \mathrm{L}$ aliquots containing 0 to $200 \mathrm{nM}$ rivaroxaban or apixaban were added to wells containing $10 \mu \mathrm{L}$ of $50 \mathrm{nM}$ factor Xa. Residual factor Xa activity was then measured by adding S-2765 and $\mathrm{CaCl}_{2}$ to a final volume of $100 \mu \mathrm{L}$, yielding final concentrations of $400 \mu \mathrm{M}$ and $5 \mathrm{mM}$, respec- tively. Absorbance was monitored at $405 \mathrm{~nm}$ using a SpectraMax Plus plate reader (Molecular Devices, Sunnyvale, California, United States) and the rates of S-2765 hydrolysis were converted to factor Xa concentrations using a specific activity of $25.7 \mathrm{mOD} \cdot \mathrm{min}^{-1} \cdot \mathrm{nM}^{-1}$, which was determined in a separate experiment (not shown). Residual concentrations of factor Xa were then plotted against anticoagulant concentrations, and the data were analyzed as above to obtain the $\mathrm{K}_{\mathrm{i}}$ values. All inhibition experiments were conducted at $25^{\circ} \mathrm{C}$ in HBST containing $0.1 \%$ Prionex and $5 \mathrm{mM} \mathrm{CaCl}$ (HBSTC). Stock solutions of inhibitors were diluted in HBST, such that the final concentration of DMSO was $<0.01 \%(\mathrm{v} / \mathrm{v})$; a concentration that, in control experiments, did not influence the hydrolysis of S-2765 by factor Xa.

\section{Rate Constants of Inhibition of Factor Xa}

The rates of association and dissociation of the inhibitors with factor Xa were determined simultaneously in HBSTC buffer under pseudo first-order conditions using Molecular Devices Flex Station 3 plate reader. In microplate wells, $100-\mu \mathrm{L}$ aliquots of a solution containing the inhibitor and Pefafluor Xa were added to $100-\mu \mathrm{L}$ aliquots of factor Xa, such that final concentrations were $2.5 \mathrm{nM}, 100 \mu \mathrm{M}$, and $0.25 \mathrm{nM}$, respectively. Samples were rapidly mixed by the instrument and fluorescence was monitored at 2-second intervals at $37^{\circ}$ $C$ with excitation and emission wavelengths of 342 and $440 \mathrm{~nm}$, respectively, and a 435-nm cutoff filter. To convert relative fluorescence units to nominal concentrations of 7-amino 4-methylcoumarin (AMC), a standard curve was generated from intensity values at $440 \mathrm{~nm}\left(\mathrm{I}_{440}\right)$ of varying concentrations of fully hydrolyzed Pefafluor Xa.

For analysis of the results, a mixed inhibition model ${ }^{10}$ was fit to each time course of AMC production ( - Scheme 1) using equations (see - Supplementary Materials) formulated to determine the best-fit individual rate constants of association and dissociation by numerical integration using Berkeley Madonna Software (University of California, Berkeley, California, United States). ${ }^{21}$ In this model, FXa is the free factor Xa concentration, $S$ is the concentration of Pefafluor $\mathrm{Xa}$, I is the concentration of inhibitor (apixaban or rivaroxaban), FXa.S is the factor Xa-Pefafluor Xa complex, FXa.I is the factor Xa-inhibitor complex, and FXa.S.I is the ternary

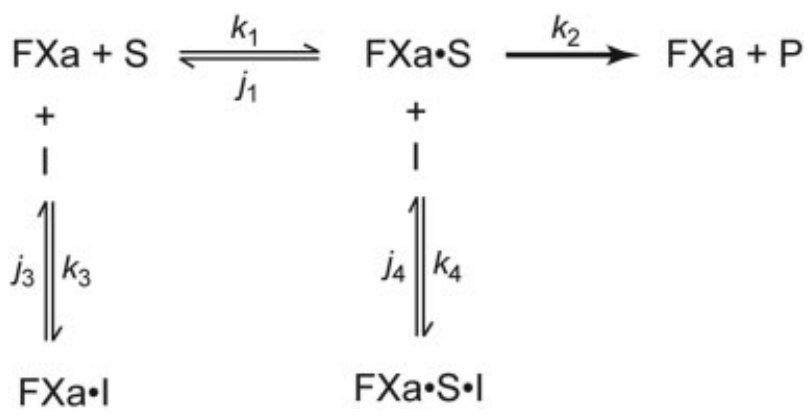

Scheme 1 Mixed inhibition of factor Xa by apixaban or rivaroxaban. This model depicts two ways in which factor $X$ a may bind its inhibitors. FXa, factor Xa; S, substrate (Pefafluor Xa); I, inhibitor (apixaban or rivaroxaban). 
factor Xa-Pefafluor Xa-inhibitor complex. The best-fit rate constants were first determined in the absence of inhibitors, then the theoretical dissociation constants for each inhibitor were calculated for free factor $\mathrm{Xa}\left(\mathrm{K}_{\mathrm{Xa} 1}=j_{3} / k_{3}\right)$ and for the factor Xa-substrate complex $\left(\mathrm{K}_{\mathrm{Xa} 2}=j_{4} / k_{4}\right)$.

\section{Rate Constants of Inhibition of Factor Xa in Prothrombinase}

The individual rate constants for association and dissociation of the inhibitors with factor Xa incorporated into the prothrombinase complex were determined by measuring thrombin generation to infer residual factor Xa activity in real time. Thrombin generation was quantified using DAPA, a probe that specifically inhibits thrombin and exhibits increased fluorescence on doing so. ${ }^{29}$ For the prothrombinase reaction, $60 \mu \mathrm{L}$ of a $2 \times$ stock solution (solution A) containing $1 \mathrm{nM}$ factor $\mathrm{Xa}$, $11 \mathrm{nM}$ factor Va, $50 \mu \mathrm{M}$ PCPS, and $10 \mu \mathrm{M}$ DAPA was added to wells of a 96-well UV-compatible microtiter plate (Corning). In a separate low protein-binding 96-well plate (Thermo), $80 \mu \mathrm{L}$ of a $2 \times$ solution (solution B) containing $1 \mu \mathrm{M}$ prothrombin and varying concentrations of either apixaban or rivaroxaban $(0,2$, 10 , or $30 \mathrm{nM}$ ) was added. All reagents were dissolved in $20 \mathrm{mM}$ Tris- $\mathrm{HCl}, 0.15 \mathrm{M} \mathrm{NaCl}, \mathrm{pH} 7.4$, and $5 \mathrm{mM} \mathrm{CaCl}_{2}$. Both plates were then placed in a FlexStation 3 (Molecular Devices) and allowed to equilibrate at $37^{\circ} \mathrm{C}$ for 5 minutes and fluorescence was monitored by reading from the bottom of the wells at 2second intervals using excitation and emission wavelengths of 280 and $545 \mathrm{~nm}$, respectively, and a $530 \mathrm{~nm}$ cutoff filter. After determining baseline fluorescence for 30 seconds, $60-\mu \mathrm{L}$ aliquots of solution $B$ were then transferred to the respective wells of the first plate, and samples were mixed by instrument trituration. Experiments were performed at least three times. To convert the fluorescence signal to nominal thrombin con- centration, a standard curve was generated using known amounts of thrombin in the presence of DAPA.

For analysis, data were fit using a mixed inhibition model $^{10}$ (-Scheme 2), wherein all forms of factor Xa (E), both free or within prothrombinase, could interact with the inhibitor (I). Prothrombin (P) activation by prothrombinase was limited to the meizothrombin (M) pathway. This approach is justified because (1) meizothrombin is the predominant intermediate generated when prothrombin is activated by factor Xa in the presence of factor Va; (2) there was minimal prethrombin-2 accumulation by SDS-PAGE analysis (data not shown); and (3) unlike meizothrombin or thrombin, prethrombin-2 does not possess a functional active site. ${ }^{30}$ Therefore, the change in fluorescence signal can be attributed to thrombin or meizothrombin. Ratcheting of meizothrombin, a phenomenon whereby meizothrombin alters its conformation and renders the second cleavage by prothrombinase more effectively, was included $\left(k_{5}, j_{5}\right.$ in -Scheme 2) to be consistent with current models of prothrombinase. ${ }^{31}$ The inhibition rate constants for the binding of the inhibitors to prothrombinase when it is in complex with either form of meizothrombin were kept the same. In addition, the fluorescence quantum yield of meizothrombin was taken to be 1.5 times that of thrombin. ${ }^{32,33}$ Based on this model, 13 differential equations for the reactants and product were formulated (see - Supplementary Materials) to determine the best-fit rate constants as described earlier. Briefly, the rate constants of the main reaction that do not involve the inhibitor $\left(k_{\mathrm{x}}\right.$ and $\left.j_{\mathrm{x}}\right)$ were determined first using similar step-wise restrictions imposed over rounds of fitting (-Supplementary Table S1). Once the best-fit $k_{\mathrm{x}}$ and $j_{\mathrm{x}}$ values for each set of data were obtained, they were fixed in order to determine the best-fit $k i_{\mathrm{x}}$ and $j i_{\mathrm{x}}$ values for each inhibitor. The

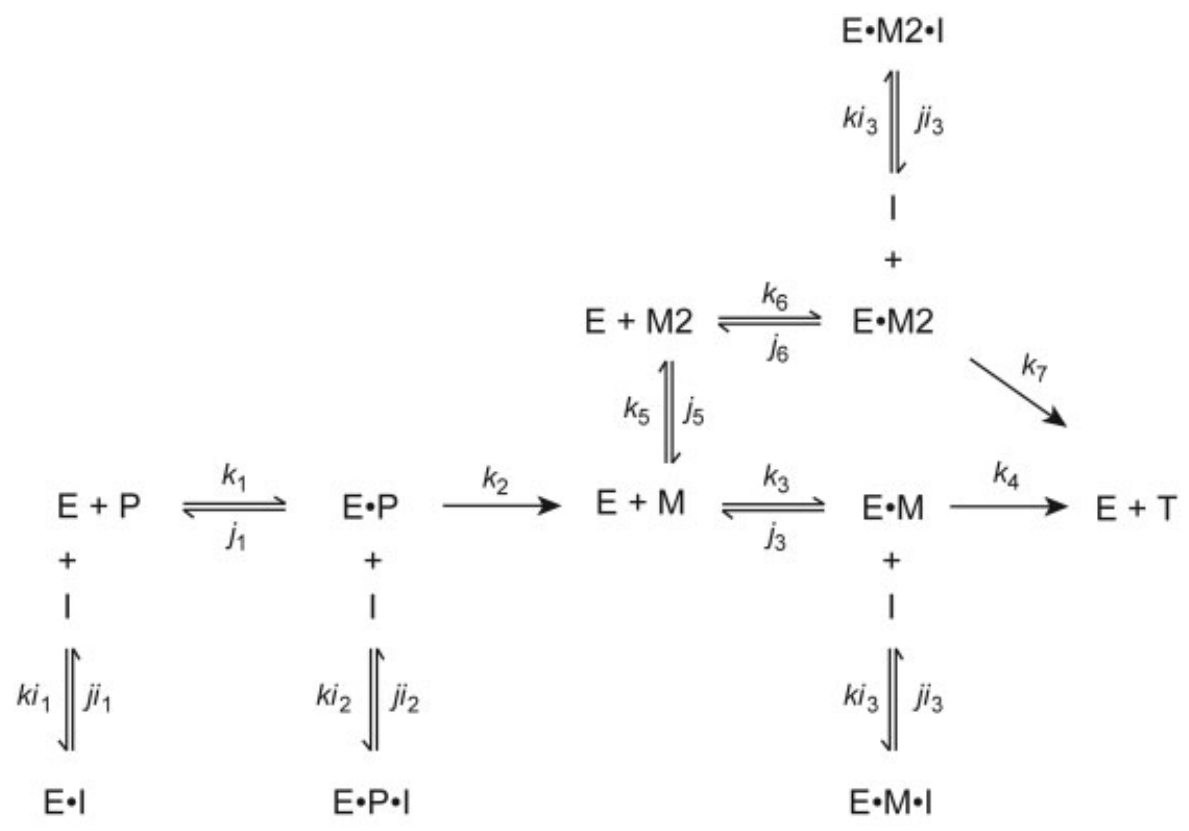

Scheme 2 Mixed inhibition of factor Xa in prothrombinase by apixaban or rivaroxaban during prothrombin activation. This model depicts three ways in which prothrombinase may bind its inhibitors. Ratcheting is included in this model, which signifies a conformational change of the intermediate, meizothrombin, so that it is able to be further processed to thrombin. E, prothrombinase; P, prothrombin; M, meizothrombin; M2, ratcheted meizothrombin; T, thrombin; I, inhibitors (apixaban or rivaroxaban). 
theoretical dissociation constants for each inhibitor were then calculated for prothrombinase without prothrombin $\left(\mathrm{K}_{\text {Pase } 1}=j i_{1} / k i_{1}\right)$, prothrombinase in complex with prothrombin $\left(\mathrm{K}_{\mathrm{Pase} 2}=j i_{2} / k i_{2}\right)$, and for prothrombinase in complex with either form of meizothrombin $\left(\mathrm{K}_{\text {Pase } 3}=j i_{3} / k i_{3}\right)$ using the individual on- and off-rates.

To determine whether the divergent effects of rivaroxaban and apixaban on the coagulation assays are due to differential inhibition of factor Xa leading to differences in substrate recognition, a mutated form of prothrombin $\mathrm{rMZ}$, which can only be cleaved at Arg320, was used as the substrate so that initial cleavage at this site could be examined in isolation. ${ }^{34}$ rMZ activation by prothrombinase was monitored and the data were analyzed under conditions identical to those used with wild-type prothrombin. The model shown in - scheme 1 was used to describe this reaction, where the substrate (S) was replaced with $\mathrm{rMZ}$ and the product $(\mathrm{P})$ was replaced with rMZa. The theoretical dissociation constants for rivaroxaban or apixaban were again calculated using the best-fit rate constants determined as described earlier.

\section{Statistical Analyses}

All experiments were performed at least in triplicate and data are expressed as mean \pm SD. Inhibition constants $\left(\mathrm{K}_{\mathrm{i}}\right)$ and inhibition rates $\left(k_{2}\right)$ for rivaroxaban and apixaban were compared by Student's $t$-tests using Microsoft Office Excel 2010. PT, aPTT, and thrombin generation data were compared by two-way analysis of variance using GraphPad Prism 6 (San Diego, California, United States). For all analyses, $p$ values $<0.05$ were considered statistically significant.

\section{Results}

\section{Effect of Inhibitors on the PT and aPTT}

The effects of rivaroxaban and apixaban on the PT and aPTT were examined using concentrations up to 2,530 and $2,350 \mu \mathrm{M}(1,100 \mathrm{ng} / \mathrm{mL})$, respectively, well beyond the peak plasma levels obtained with the doses of the drugs that are used clinically. ${ }^{26,35}$ Although both agents prolonged the PT and aPTT in a concentration-dependent manner (-Fig. 1), rivaroxaban had a significantly $(p<0.0001)$ greater effect on both tests than apixaban, consistent with previous reports. ${ }^{13,27}$ Therefore, rivaroxaban has a greater effect on global tests of coagulation than apixaban, regardless of whether coagulation is activated via the extrinsic or intrinsic pathway.

\section{Plasma-Based Thrombin Generation Assay}

The inhibitory effects of rivaroxaban and apixaban on thrombin generation induced by $3 \mathrm{pM}$ tissue factor in plasma were compared using a calibrated thrombin generation assay. Both agents prolonged the lag time, reduced peak thrombin concentration, and decreased endogenous thrombin potential (ETP) in a concentration-dependent manner (-Fig. 2). Rivaroxaban significantly prolonged the lag time and decreased ETP compared with apixaban, consistent with previous reports. ${ }^{19,20}$ The agents had similar effects on other parameters of thrombin generation.
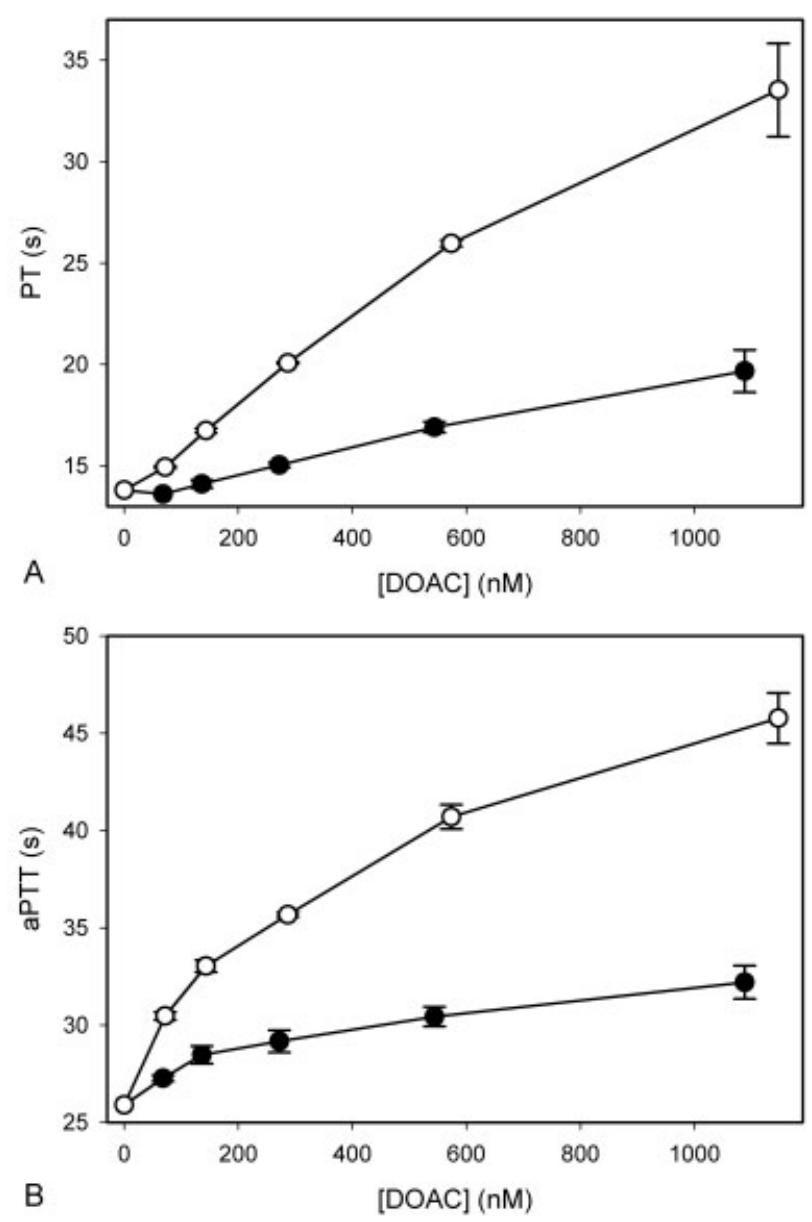

Fig. 1 Comparison of the effects of rivaroxaban and apixaban on the prothrombin time (PT) and activated partial thromboplastin time (aPTT). The (A) PT and (B) aPTT were determined in human plasma in the presence of increasing concentrations of apixaban (closed) or rivaroxaban (open). Symbols reflect the mean \pm SD of four experiments.

\section{Inhibition of Factor $X a$}

To begin to investigate the mechanism responsible for the divergent effects of rivaroxaban and apixaban, we first measured their steady-state binding affinities for free factor $\mathrm{Xa}$ and for factor Xa assembled in the prothrombinase complex using a chromogenic assay for factor Xa. Rivaroxaban and apixaban inhibited the chromogenic activity of free factor Xa in a concentration-dependent manner (-Fig. 3) with $\mathrm{K}_{\mathrm{i}}$ values of $2.6 \pm 0.4 \mathrm{nM}$ and $2.0 \pm 0.1 \mathrm{nM}$, respectively, values comparable with those previously reported. ${ }^{9,10}$ At a concentration of $20 \mathrm{nM}$, both agents inhibited the chromogenic activity of $5 \mathrm{nM}$ factor Xa by $\sim 85 \%$.

Studies of factor Xa inhibition were then repeated in the presence of factor Va, PCPS, and prothrombin. Hirudin was added to prevent substrate hydrolysis by the generated thrombin. As observed with factor Xa alone, in the presence of prothrombinase components, rivaroxaban and apixaban inhibited over $95 \%$ of factor Xa chromogenic activity in the same dose-dependent manner (not shown) with $\mathrm{K}_{\mathrm{i}}$ values of $1.0 \pm 0.6 \mathrm{nM}$ and $0.9 \pm 0.8 \mathrm{nM}$, respectively, values not significantly different from those determined with free factor Xa. Together, these data confirm that rivaroxaban and 

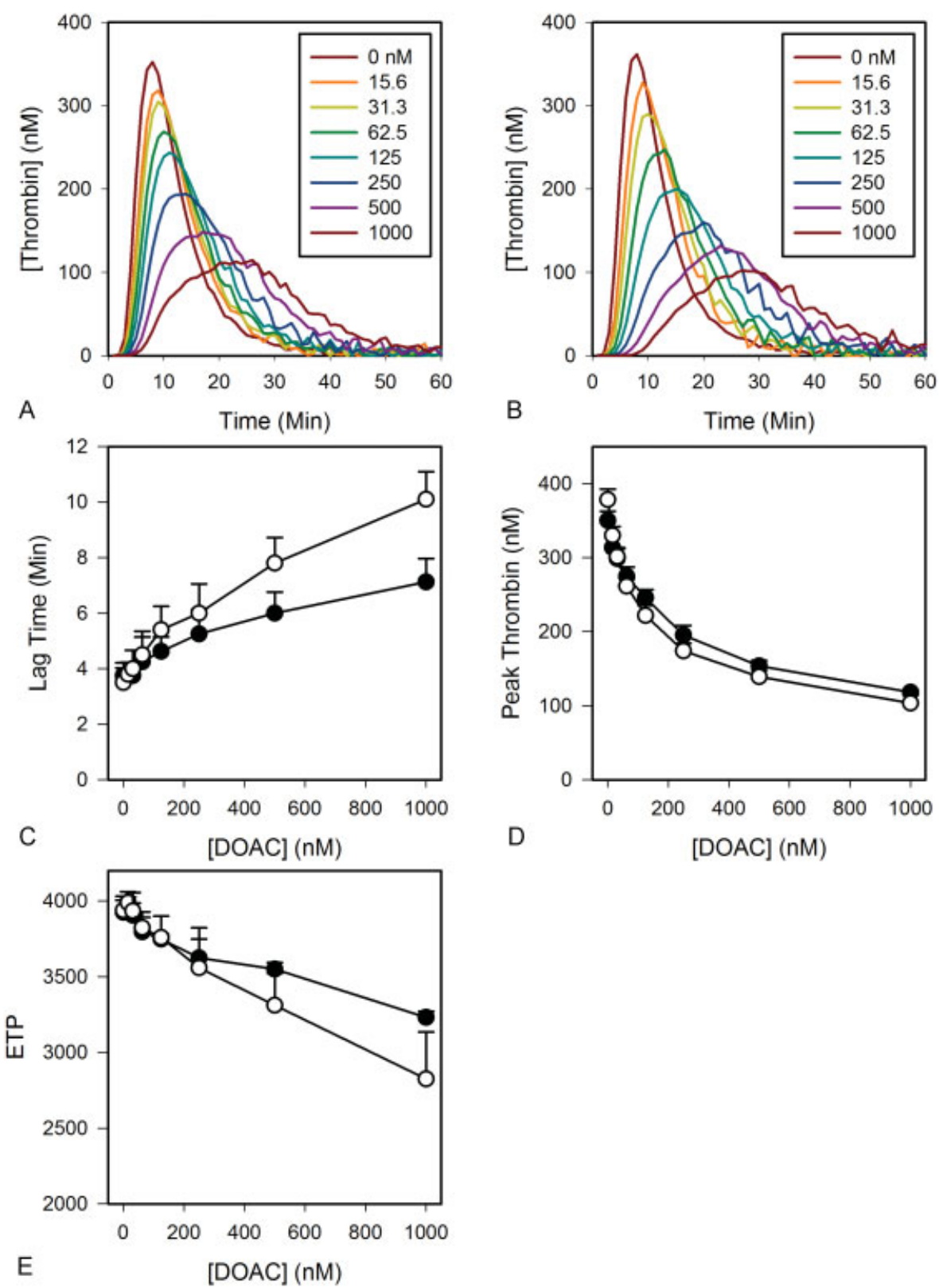

Fig. 2 Comparison of the effects of apixaban and rivaroxaban on thrombin generation in plasma. Thrombin generation in plasma containing $4 \mu \mathrm{M}$ PCPS was triggered by addition of $3 \mathrm{pM}$ tissue factor and $7.5 \mathrm{mM} \mathrm{CaCl}_{2}$ and quantified by monitoring the hydrolysis of $0.5 \mathrm{mM} Z-G l y-G l y-$ Arg-AMC in the absence or presence of (A) apixaban, or (B) rivaroxaban, at the indicated concentrations. Using the thrombin generation profiles, (C) lag time, (D) peak thrombin concentration, and (E) ETP are measured and plotted versus the concentrations of apixaban (closed) or rivaroxaban (open). Data reflect the mean \pm SD of three experiments. ETP, endogenous thrombin potential.

apixaban bind to free factor Xa and factor Xa incorporated into the prothrombinase complex with comparable affinities. ${ }^{9,10}$ Therefore, differences in steady-state binding affinities do not explain the divergent effects of rivaroxaban and apixaban on the PT, aPTT, and thrombin generation.

\section{Inhibition of Prothrombinase-Induced Thrombin Generation}

The previous experiments examined the effect of the inhibitors on factor Xa chromogenic activity, an interaction that excludes those employed by prothrombin at sites remote from the active site. ${ }^{36}$ To examine the effect of the inhibitors when prothrombin is the substrate of prothrombinase, we quantified thrombin generation. Although both inhibitors reduced prothrombinase-induced thrombin production in a concentration-dependent manner ( - Fig. 4 ), the $K_{i}$ value for rivaroxaban was 4-fold lower than that for apixaban $(0.7 \pm 0.3 \mathrm{nM}$ and $2.9 \pm 0.5 \mathrm{nM}$, respectively; $p<0.02)$. Therefore, rivaroxaban is a more potent inhibitor of prothrombinase-induced thrombin formation than apixaban.

\section{Inhibition Rate Constants: Factor Xa Binding}

To better understand why rivaroxaban and apixaban have different effects on prothrombinase-induced thrombin generation, we next compared the binding kinetics of the inhibitors with factor Xa using a mixed inhibition model 


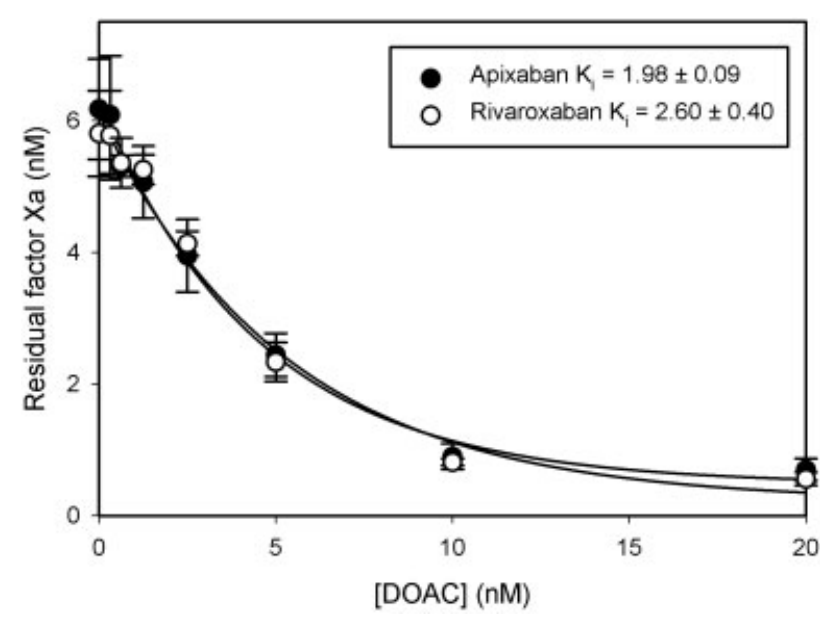

Fig. 3 Comparison of the effects of rivaroxaban and apixaban on factor Xa chromogenic activity. Inhibition constants $\left(\mathrm{K}_{\mathrm{i}}\right)$ were determined by incubating $5 \mathrm{nM}$ factor $\mathrm{Xa}$ with rivaroxaban (open) or apixaban (closed) at the indicated concentrations, and residual active factor Xa concentration was determined by monitoring hydrolysis of $500 \mu \mathrm{M}$ S-2765. Symbols reflect the mean \pm SD of three to four experiments. Lines are fit to a rectangular hyperbola equation by nonlinear regression analysis.

(-Scheme 1)..$^{10}$ Inhibition was quantified by continuously monitoring factor Xa fluorogenic activity in the presence of rivaroxaban or apixaban. Although the time courses revealed gradual reduction of factor Xa fluorogenic activity, as evidenced by declining rates of fluorophore release with both inhibitors (-Fig. 5), plots of residual factor Xa activity revealed more rapid inhibition with rivaroxaban than with apixaban (-Fig. 5, inset). Plots of product versus time were analyzed by global fitting and the best-fit values for $k_{1}, j_{1}$, and

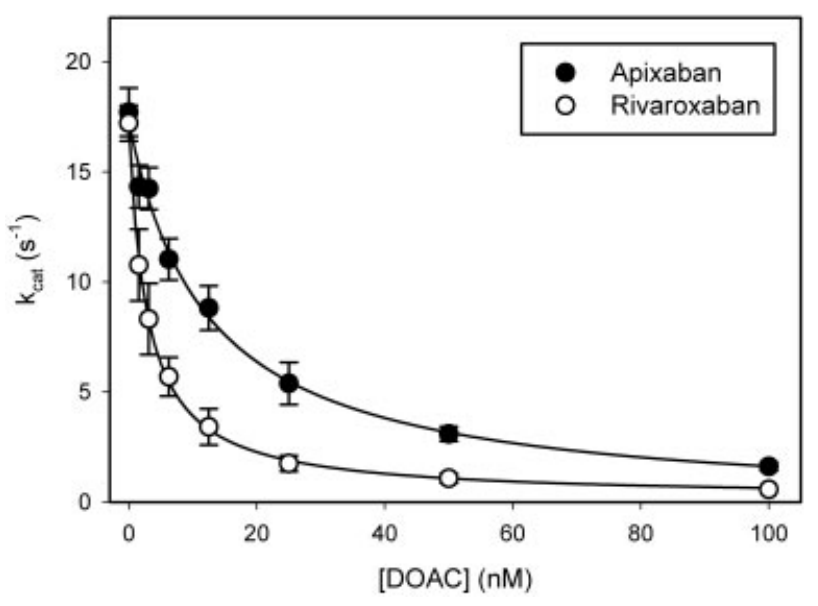

Fig. 4 Comparison of the effects of rivaroxaban and apixaban on prothrombin activation by prothrombinase. Inhibition constants $\left(K_{i}\right)$ were determined by incubating increasing concentrations of rivaroxaban (open) or apixaban (closed) with $0.5 \mathrm{nM}$ factor $\mathrm{Xa}, 5.5 \mathrm{nM}$ factor Va, $50 \mu \mathrm{M}$ PCPS, $500 \mathrm{nM}$ prothrombin, and $5 \mathrm{mM} \mathrm{CaCl}_{2}$. After $20 \mathrm{~s}$, reactions were stopped by addition of EDTA to $10 \mathrm{mM}$ and generated thrombin was quantified by chromogenic assay with ChzTh. Rates of prothrombin activation are plotted versus inhibitor concentration. Symbols reflect the mean \pm SD of three to four experiments. Lines are fit to a rectangular hyperbola equation by nonlinear regression analysis.

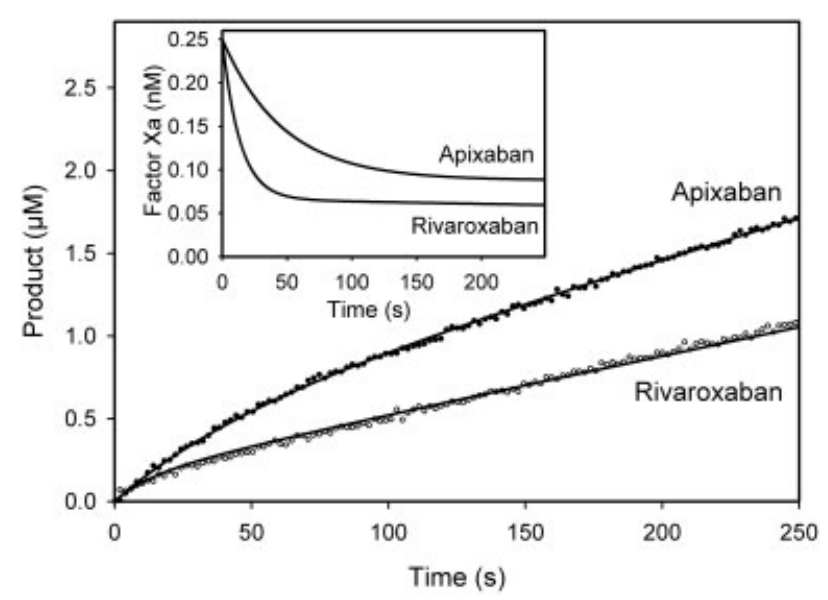

Fig. 5 Inhibition of factor Xa amidolytic activity in the presence of apixaban and rivaroxaban. Cleavage of $100 \mu \mathrm{M}$ Pefafluor Xa by $0.25 \mathrm{nM}$ factor Xa was monitored in a fluorescent plate reader at 2-s intervals. Reactions were performed at $37^{\circ} \mathrm{C}$ in HBSTC containing $2.5 \mathrm{nM}$ apixaban (closed) or rivaroxaban (open). Fluorescence intensity values were converted to concentration of 4-amino methyl coumarin (product) and plotted versus time. Data were fit to a mixed inhibition model (lines). The inset shows nominal residual factor $\mathrm{Xa}$ concentration determined from the fitted data plotted versus time.

$k_{2}$ were determined to be $7.05 \pm 0.30 \mathrm{nM}^{-1} \cdot \mathrm{s}^{-1}, 1.74 \pm 0.09$ $\mathrm{s}^{-1}$, and $187 \pm 14 \mathrm{~s}^{-1}$, respectively. With these values fixed, the best-fit values for the rate constants for inhibition were determined (-Table $\mathbf{1}$ ). The on- and off-rate constants for binding factor Xa alone ( $k_{3}$ and $j_{3}$, respectively) for rivaroxaban were, on average, 21-fold faster than those for apixaban. A similar trend was observed for inhibitor binding to the factor Xa-fluorogenic substrate complex $\left(k_{4}\right.$ and $\left.j_{4}\right)$, whereby rivaroxaban bound, on average, 25 -fold faster than apixaban. Therefore, contrary to a previous report, ${ }^{19}$ our data suggest that a faster on-rate is not the sole explanation for the greater effect of rivaroxaban on tests of coagulation than apixaban.

\section{Inhibition Rate Constants: Prothrombin Activation}

Because of the differences observed in the prothrombinase endpoint assay (-Fig. 4), we used a continuous assay to determine the kinetic rate constants for inhibition of factor Xa assembled in prothrombinase. Thrombin generation was quantified by continuously monitoring the increase in fluorescence that accompanies binding of DAPA, a fluorophore that binds exclusively to the active site of meizothrombin or thrombin. ${ }^{29}$ Plots of thrombin concentration versus time in the presence of varying concentrations of the inhibitors were analyzed using the mixed inhibition model (-Scheme 2), wherein the inhibitor is allowed to bind to prothrombinasebound factor Xa in the absence or presence of prothrombin or meizothrombin. Concentrations of thrombin and meizothrombin over time were calculated by numerical integration. Individual rate constant parameter values were optimized using the Simplex procedure ${ }^{37}$ as described previously. ${ }^{21}$ Best-fit association and dissociation rate constants, and the calculated $K_{\text {Pase }}$ value, representing the dissociation constant for inhibitor with factor Xa in prothrombinase, are illustrated in - Table $\mathbf{2}$. The rate constants 
Table 1 Kinetic parameters of factor Xa inhibition by apixaban and rivaroxaban determined according to - Scheme 1 by nonlinear regression analyses of chromogenic substrate cleavage data

\begin{tabular}{|l|l|l|l|}
\hline \multirow{2}{*}{ Constant } & Inhibitor & Rivaroxaban & \multirow{2}{*}{ Fold } \\
\cline { 2 - 4 } & Apixaban & $(3.77 \pm 0.32) \times 10^{-2}$ & 10.3 \\
\hline$k_{3}\left(\mathrm{nM}^{-1} \cdot \mathrm{s}^{-1}\right)$ & $(3.65 \pm 0.24) \times 10^{-3}$ & $(3.21 \pm 0.28) \times 10^{-2}$ & 30.9 \\
\hline$j_{3}\left(\mathrm{~s}^{-1}\right)$ & $(1.04 \pm 0.06) \times 10^{-3}$ & $(8.54 \pm 0.78) \times 10^{-1}$ & 3.0 \\
\hline $\mathrm{K}_{\mathrm{Xa} 1}(\mathrm{nM})$ & $(2.85 \pm 0.23) \times 10^{-1}$ & $(1.34 \pm 0.04) \times 10^{-2}$ & 45.4 \\
\hline$k_{4}\left(\mathrm{nM}^{-1} \cdot \mathrm{s}^{-1}\right)$ & $(2.95 \pm 0.29) \times 10^{-4}$ & $(2.01 \pm 0.17) \times 10^{-2}$ & 5.2 \\
\hline$j_{4}\left(\mathrm{~s}^{-1}\right)$ & $(3.86 \pm 0.32) \times 10^{-3}$ & $1.49 \pm 0.12$ & 0.1 \\
\hline $\mathrm{K}_{\mathrm{Xa2}}(\mathrm{nM})$ & $(1.32 \pm 0.15) \times 10^{1}$ & \\
\hline
\end{tabular}

Notes: $\mathrm{K}_{\mathrm{Xa}}$ values represent off- and on-rate quotients. Fold is the ratio between rivaroxaban and apixaban.

associated with prothrombin activation by prothrombinase in the absence of inhibitor are shown in - Supplementary Table S1. Using the best-fit rate constants, regression lines for the progress curves in the presence of 2 to $30 \mathrm{nM}$ apixaban (-Fig. 6A) or rivaroxaban (-Fig. 6B) were generated. On-rate constants $k i_{1}$ and $k i_{2}$ for rivaroxaban were 1,193-fold and 3fold higher than those for apixaban, respectively (-Table 2). The higher $k i_{1}$ and $k i_{2}$ values with rivaroxaban are comparable with the 10 -fold higher $k_{3}$ and 45 -fold higher $k_{4}$ values observed with rivaroxaban relative to apixaban in the factor Xa chromogenic assay (-Table 1). Interestingly, the on-rate for rivaroxaban with the prothrombinase-meizothrombin complex, $k i_{3}$, was 3 -fold slower than that for apixaban. Therefore, rivaroxaban and apixaban have different off-rates as well as different on-rates. The greatest difference in off:on $\left(j i_{\mathrm{x}} / k i_{\mathrm{x}}\right)$ rate ratios of these constants $\left(\mathrm{K}_{\text {Pase }}\right)$ was with free enzyme inhibition $\left(K_{\text {Pase1 }}\right)$, where the value for rivaroxaban was 454-fold lower than that for apixaban, mostly due to the very low $k i_{1}$ value for apixaban (-Table 2 ). Closer inspection reveals that only the on-rate of apixaban is altered by the assembly of factor Xa into prothrombinase, suggesting that rivaroxaban and apixaban interact with factor Xa differently.

\section{Inhibition Rate Constants: rMZ Activation}

Because prothrombin activation requires two cleavages, kinetic analyses are complicated by the requirement to account for parallel reaction pathways. To simplify the analysis, we used rMZ, a recombinant prothrombin

Table 2 Kinetic parameters of inhibition of factor Xa incorporated in prothrombinase by apixaban or rivaroxaban as measured using thrombin-DAPA fluorescence

\begin{tabular}{|c|c|c|c|c|}
\hline \multirow[t]{2}{*}{ Reaction } & \multirow[t]{2}{*}{ Constant } & \multicolumn{2}{|l|}{ Inhibitor } & \multirow[t]{2}{*}{ Fold } \\
\hline & & Apixaban & Rivaroxaban & \\
\hline \multirow[t]{3}{*}{$\mathrm{E}+\mathrm{I} \Leftrightarrow \mathrm{E} \cdot \mathrm{I}$} & $\begin{array}{l}k i_{1} \\
\left(\mathrm{nM}^{-1} \cdot \mathrm{s}^{-1}\right)\end{array}$ & $(4.51 \pm 0.02) \times 10^{-6}$ & $(5.38 \pm 0.35) \times 10^{-3}$ & 1192.9 \\
\hline & $\begin{array}{l}j i_{1} \\
\left(\mathrm{~s}^{-1}\right)\end{array}$ & $(4.99 \pm 0.05) \times 10^{-4}$ & $(1.31 \pm 0.11) \times 10^{-3}$ & 2.6 \\
\hline & $\begin{array}{l}K_{\text {Pase } 1} \\
(\mathrm{nM})\end{array}$ & $(1.11 \pm 0.02) \times 10^{2}$ & $(2.44 \pm 0.31) \times 10^{-1}$ & 0.002 \\
\hline \multirow[t]{3}{*}{$E \cdot P+I \Leftrightarrow E \cdot P \cdot I$} & $\begin{array}{l}k i_{2} \\
\left(\mathrm{nM}^{-1} \cdot \mathrm{s}^{-1}\right)\end{array}$ & $(4.30 \pm 0.18) \times 10^{-3}$ & $(1.24 \pm 0.02) \times 10^{-2}$ & 2.9 \\
\hline & $\begin{array}{l}j i_{2} \\
\left(s^{-1}\right)\end{array}$ & $(5.81 \pm 0.30) \times 10^{-3}$ & $(1.12 \pm 0.15) \times 10^{-2}$ & 1.9 \\
\hline & $\begin{array}{l}K_{\text {Pase2 }} \\
(\mathrm{nM})\end{array}$ & $1.35 \pm 0.05$ & $(8.99 \pm 1.16) \times 10^{-1}$ & 0.7 \\
\hline \multirow{3}{*}{$\begin{array}{l}\mathrm{E} \cdot \mathrm{M}+\mathrm{I} \Leftrightarrow \mathrm{E} \cdot \mathrm{M} \cdot \mathrm{I} \\
\text { or } \\
\mathrm{E} \cdot \mathrm{M} 2+\mathrm{I} \Leftrightarrow \mathrm{E} \cdot \mathrm{M} 2 \cdot \mathrm{I}\end{array}$} & $\begin{array}{l}k i_{3} \\
\left(\mathrm{nM}^{-1} \cdot \mathrm{s}^{-1}\right)\end{array}$ & $(6.20 \pm 0.33) \times 10^{-3}$ & $(2.37 \pm 0.22) \times 10^{-3}$ & 0.4 \\
\hline & $\begin{array}{l}j i_{3} \\
\left(\mathrm{~s}^{-1}\right)\end{array}$ & $(2.64 \pm 0.19) \times 10^{-5}$ & $(2.95 \pm 0.11) \times 10^{-3}$ & 111.7 \\
\hline & $\begin{array}{l}K_{\text {Pase3 }} \\
(\mathrm{nM})\end{array}$ & $(4.28 \pm 0.01) \times 10^{-1}$ & $1.25 \pm 0.16$ & 2.9 \\
\hline
\end{tabular}

Notes: $K_{\text {Pase }}$ values represent off- and on-rate quotients. Fold is the ratio between rivaroxaban and apixaban. 

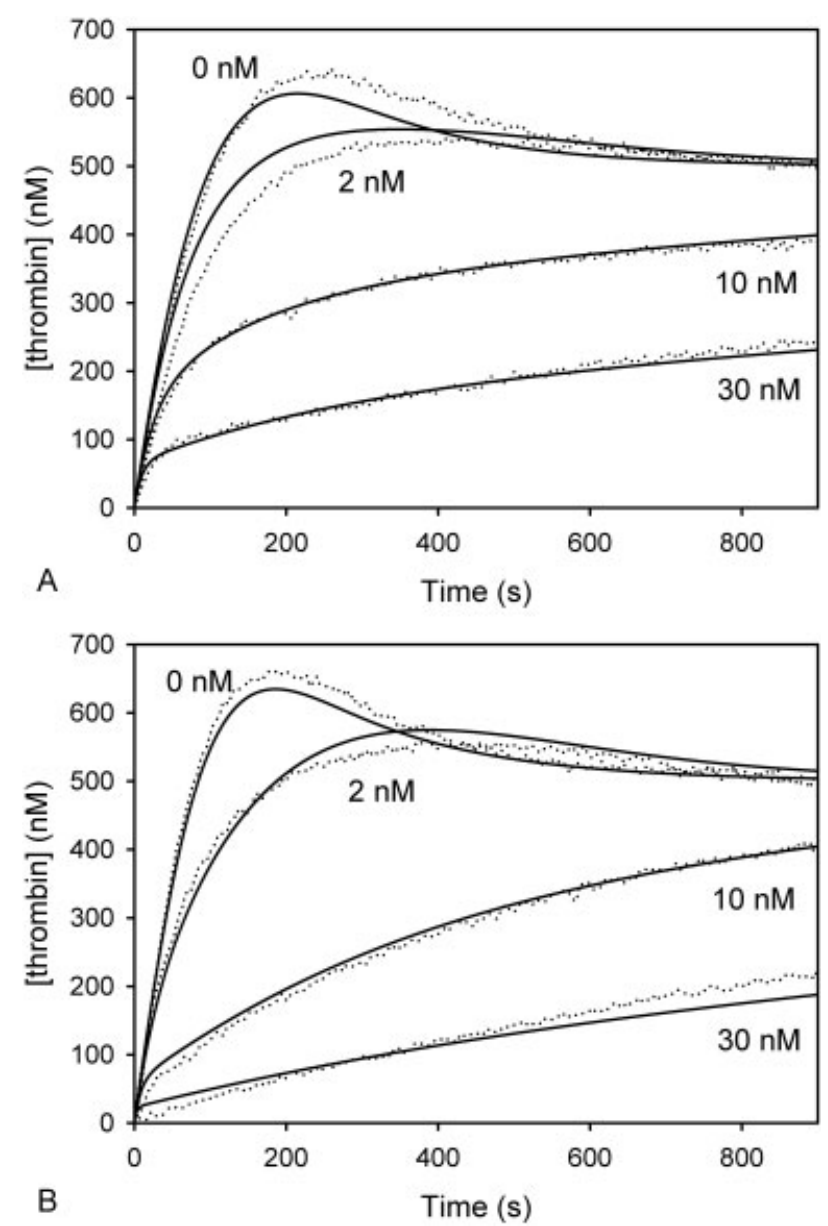

Fig. 6 Inhibition of factor $X a$ assembled in the prothrombinase complex by the presence of apixaban and rivaroxaban. Activation of $500 \mathrm{nM}$ prothrombin by $0.5 \mathrm{nM}$ prothrombinase $(0.5 \mathrm{nM}$ factor $\mathrm{Xa}$, $5 \mathrm{nM}$ factor $\mathrm{Va}, 50 \mu \mathrm{M}$ PCPS, $5 \mathrm{mM} \mathrm{CaCl}$ ) containing (A) apixaban or (B) rivaroxaban at varying concentrations $(2,10$, or $30 \mathrm{nM})$ was monitored using DAPA in a fluorescent plate reader at $37^{\circ} \mathrm{C}$. Thrombin generation was monitored at 2-s intervals. Data were fit to a mixed inhibition model (lines). derivative that can only be cleaved at Arg320. ${ }^{34}$ Activation of rMZ was monitored using DAPA and kinetic analyses were performed as outlined earlier. Best-fit rate constants ( - Table 3 ) were used to generate regression lines ( - Fig. 7). The off:on $\left(j i_{\mathrm{x}} / k i_{\mathrm{x}}\right)$ ratio of free prothrombinase $\left(K_{\text {Pase1 }}\right)$ was 16 -fold lower with rivaroxaban than with apixaban, whereas $\mathrm{K}_{\mathrm{Pase} 2}$, representing inhibition of the prothrombinase-rMZ complex, was 3-fold lower with rivaroxaban than with apixaban. The difference in the effect between rivaroxaban and apixaban on rMZ activation is most evident in the initial stages of rMZ activation (-Fig. 7, inset), similar to what was observed with wild-type prothrombin (-Fig. 6). Therefore, these data suggest that rivaroxaban and apixaban have different effects on the initial interaction of factor Xa in the prothrombinase complex with prothrombin, and that this difference occurs upon the initial presentation and cleavage of prothrombin at Arg320.

\section{Discussion}

Previous studies have shown that rivaroxaban has a greater effect on the PT and aPTT than on apixaban. ${ }^{12-14,17}$ To determine whether the differences between the on-rates of the inhibitors for free factor $\mathrm{Xa}^{19}$ are maintained when factor Xa is incorporated into prothrombinase and to correlate those with global tests of coagulation, we compared the effects of rivaroxaban and apixaban in three different systems. First, we compared their effects in plasma on the PT, aPTT, and thrombin generation assay. Second, we determined their $K_{i}$ values for free factor Xa and factor Xa incorporated into prothrombinase. Third, we measured the rate constants for inhibition of factor Xa alone and when assembled in prothrombinase.

As previously reported, rivaroxaban and apixaban inhibited factor Xa with similar $\mathrm{K}_{\mathrm{i}}$ values, confirming that the agents bind factor $\mathrm{Xa}$ with similar affinities at equilibrium. $^{9,10,19}$ Also consistent with previous results, at

Table 3 Kinetic parameters of factor Xa inhibition by apixaban or rivaroxaban as measured using meizothrombin-DAPA fluorescence

\begin{tabular}{|c|c|c|c|c|}
\hline \multirow[t]{2}{*}{ Reaction } & \multirow[t]{2}{*}{ Constant } & \multicolumn{2}{|l|}{ Inhibitor } & \multirow[t]{2}{*}{ Fold } \\
\hline & & Apixaban & Rivaroxaban & \\
\hline \multirow[t]{3}{*}{$\mathrm{E}+\mathrm{I} \Leftrightarrow \mathrm{E} \cdot \mathrm{I}$} & $\begin{array}{l}k i_{1} \\
\left(\mathrm{nM}^{-1} \cdot \mathrm{s}^{-1}\right)\end{array}$ & $(4.70 \pm 0.39) \times 10^{-3}$ & $(3.46 \pm 0.49) \times 10^{-2}$ & 7.4 \\
\hline & $\begin{array}{l}j i_{1} \\
\left(\mathrm{~s}^{-1}\right)\end{array}$ & $(4.74 \pm 0.23) \times 10^{-2}$ & $(2.15 \pm 0.18) \times 10^{-3}$ & 0.05 \\
\hline & $\begin{array}{l}\text { Kase1 } \\
(\mathrm{nM})\end{array}$ & $(1.01 \pm 0.12) \times 10^{1}$ & $(6.24 \pm 0.41) \times 10^{-2}$ & 0.006 \\
\hline \multirow[t]{3}{*}{$\mathrm{E} \cdot \mathrm{rMZ}+\mathrm{I} \Leftrightarrow \mathrm{E} \cdot \mathrm{rMZ} \cdot \mathrm{I}$} & $\begin{array}{l}k i_{2} \\
\left(\mathrm{nM}^{-1} \cdot \mathrm{s}^{-1}\right)\end{array}$ & $(1.88 \pm 0.14) \times 10^{-2}$ & $(6.41 \pm 0.44) \times 10^{-3}$ & 0.3 \\
\hline & $\begin{array}{l}j i_{2} \\
\left(\mathrm{~s}^{-1}\right)\end{array}$ & $(1.02 \pm 0.10) \times 10^{-3}$ & $(1.23 \pm 0.08) \times 10^{-4}$ & 0.1 \\
\hline & $\begin{array}{l}\mathrm{K}_{\text {Pase2 }} \\
(\mathrm{nM})\end{array}$ & $(5.44 \pm 0.42) \times 10^{-2}$ & $(1.93 \pm 0.25) \times 10^{-2}$ & 0.4 \\
\hline
\end{tabular}

Notes: $K_{\text {Pase }}$ values represent off- and on-rate quotients. Fold is the ratio between rivaroxaban and apixaban. 


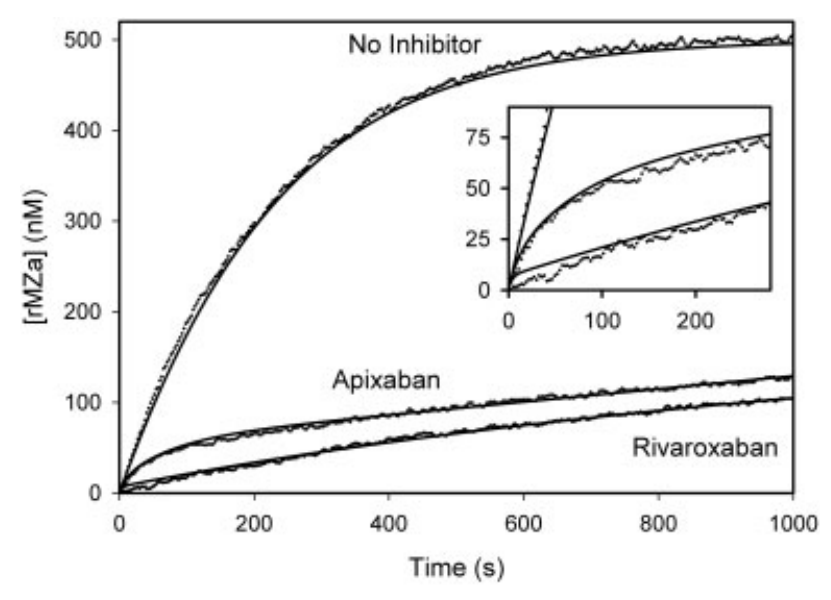

Fig. 7 rMZ was used instead of prothrombin to monitor activation cleavage at Arg320 by prothrombinase using DAPA in the absence or presence of apixaban or rivaroxaban (30 nM). rMZa generation was monitored at 2 -s intervals in a fluorescent plate reader at $37^{\circ} \mathrm{C}$. Data were fit to a mixed inhibition model (lines).

equimolar concentrations, rivaroxaban prolonged the PT and aPTT more than apixaban. ${ }^{12-14}$ In the thrombin generation assay, rivaroxaban and apixaban influenced all parameters in a concentration-dependent manner, although rivaroxaban prolonged the lag time and reduced ETP to a significantly greater extent than apixaban. Because clot formation typically occurs shortly after the lag time, the bulk of thrombin is generated after the clot has formed. The fact that rivaroxaban prolongs the lag time more than apixaban is consistent with its greater effects in clot-based assays such as PT and aPTT.

The effects of apixaban and rivaroxaban on the rates of prothrombin activation were examined. These experiments differ from those for determination of $\mathrm{K}_{\mathrm{i}}$ because all the components of prothrombinase are present, and we quantified thrombin generation rather than factor Xa activity. ${ }^{38}$ Rivaroxaban was 2-fold more potent than apixaban at inhibiting prothrombinase-induced thrombin generation. This was further investigated by determining the association and dissociation rate constants for inhibition of factor Xa. Using a mixed-type inhibition model, on- and off-rates for rivaroxaban were higher than those for apixaban. These findings are consistent with the results from the prothrombinase and clotting assays and they suggest that there are mechanistic differences in the way that rivaroxaban and apixaban interact with factor Xa incorporated within the prothrombinase complex.

rMZ was used to shed further light on the molecular mechanism responsible for the divergent inhibitory effects of rivaroxaban and apixaban on factor Xa incorporated into the prothrombinase complex. Like the results with native prothrombin, rivaroxaban is more potent than apixaban at inhibiting prothrombinase-induced activation of rMZ (-Figs. 6 and 7). Together, these data suggest that the differences between the inhibitors can mainly be attributed to their divergent inhibitory effects on the initial activation cleavage of prothrombin at Arg320 by factor Xa, which is the major pathway of prothrombin activation in the presence of factor Va, and the only available cleavage site in rMZ. The off: on ratios for rivaroxaban were 1.5 -fold (-Table 2 ) and 2.8fold ( - Table 3) lower than those for apixaban with native prothrombin and rMZ, respectively. Interestingly, incorporation of factor Xa into prothrombinase appears to have more profound effects on the inhibitory activity of apixaban than rivaroxaban, suggesting that there may be fundamental differences in the way that the two agents interact with factor Xa when it assembles into its activation complex.

A recent study suggested that the differential effects of rivaroxaban and apixaban on the PT reflected a 4-fold faster association rate constant of rivaroxaban for free factor Xa. ${ }^{19}$ We have extended this concept by examining factor Xa assembled into the prothrombinase complex. Thus, we have shown that the association rate of rivaroxaban is faster than that of apixaban not only with free factor Xa, but also with factor Xa assembled into the prothrombinase complex, and with prothrombinase activation of prothrombin. Furthermore, using the rMZ variant, we demonstrate that the initial cleavage of prothrombin at Arg320 is faster with rivaroxaban than with apixaban. Biochemical modeling of enzyme-substrate-inhibitor interaction confirms that the difference between the inhibitors is not limited to the association rates with the enzyme (either factor Xa or prothrombinase). Instead, differences in both the association and dissociation rate constants are responsible for the greater activity of rivaroxaban relative to apixaban.

Although rivaroxaban and apixaban were both developed as small molecule factor Xa inhibitors, differences in their kinetic parameters and functional potencies are evident. This could arise from differences in sites of contact with the enzyme, as well as how the substrate binds. Examination of the crystal structure of rivaroxaban and apixaban in complex with factor Xa reveals that although both factor Xa inhibitors are similarly L-shaped, ${ }^{39}$ they exhibit distinct interactions with substrate-binding subsites S1 and S4 within the active site cleft and differ in rigidity. ${ }^{40-43}$ These differences may account for the disparate association rate constants and their divergent effects on prothrombin activation.

Our study has some limitations. First, the kinetic analyses were conducted in purified systems, whereas the clotting and thrombin generation assay were performed in plasma. Therefore, translation of findings from one system to the other can be problematic. For example, binding to proteins such as albumin attenuates the anticoagulant effects in plasma. However, this phenomenon does not explain why rivaroxaban is more potent than apixaban in the plasmabased tests because apixaban exhibits less protein binding than rivaroxaban. Second, in the assays in purified systems, the binding of rivaroxaban and apixaban to factor Xa may differentially alter the kinetics of chromogenic substrate hydrolysis. ${ }^{44}$ However, the consistent differences between rivaroxaban and apixaban in all of the tests render this possibility unlikely. Therefore, there appear to be fundamental biochemical differences in the way that rivaroxaban and apixaban interact with factor Xa incorporated within the prothrombinase complex. 
In conclusion, we show that rivaroxaban is a more potent inhibitor of prothrombin activation because it inhibits factor Xa incorporated into prothrombinase more rapidly than apixaban. This difference provides an explanation for the observation that rivaroxaban has a greater effect on global tests of coagulation than apixaban.

\section{Authors' Contributions}

P.Y.K designed experiments, performed modeling and data analysis, and wrote the manuscript. C.H.Y. and B.J. D. designed and performed experiments, analyzed and interpreted data, and wrote the manuscript. B.A.L. and A. R.S. performed experiments. J.C.F., J.H., and J.I.W. designed experiments, interpreted data, and wrote the manuscript.

\section{Conflicts of Interest}

J.I.W. has served as a consultant and has received honoraria from Bristol-Myers Squibb, Pfizer, Bayer, and Janssen. None of the other authors declare conflicts of interest.

\section{Funding}

C.H.Y. is supported by a doctoral scholarship from the Canadian Institutes of Health Research. B.J.D is supported by a Scientific Education Grant from the Australasian Society of Thrombosis and Haemostasis. P.Y.K. is supported by the Hamilton Health Sciences Early Career Award and the New Investigator Fund. J.I.W. holds the Canada Research Chair (Tier I) in Thrombosis and the Heart and Stroke Foundation J. Fraser Mustard Chair in Cardiovascular Research at McMaster University.

\section{References}

1 Yeh $\mathrm{CH}$, Hogg K, Weitz JI. Overview of the new oral anticoagulants: opportunities and challenges. Arterioscler Thromb Vasc Biol 2015;35(05):1056-1065

2 Patel MR, Mahaffey KW, Garg J, et al; ROCKET AF Investigators. Rivaroxaban versus warfarin in nonvalvular atrial fibrillation. $\mathrm{N}$ Engl J Med 2011;365(10):883-891

3 Granger CB, Alexander JH, McMurray JJ, et al; ARISTOTLE Committees and Investigators. Apixaban versus warfarin in patients with atrial fibrillation. N Engl J Med 2011;365(11):981-992

4 Weitz JI, Jaffer IH, Fredenburgh JC. Recent advances in the treatment of venous thromboembolism in the era of the direct oral anticoagulants. F1000 Res 2017;6:985

5 Piran S, Schulman S. Management of venous thromboembolism: an update. Thromb J 2016;14(Suppl 1):23

6 Becattini C, Agnelli G. Treatment of venous thromboembolism with new anticoagulant agents. J Am Coll Cardiol 2016;67(16): 1941-1955

7 Barnes GD, Lucas E, Alexander GC, Goldberger ZD. National trends in ambulatory oral anticoagulant use. Am J Med 2015;128(12): 1300-5.e2

8 Weitz JI, Semchuk W, Turpie AG, et al. Trends in prescribing oral anticoagulants in Canada, 2008-2014. Clin Ther 2015;37(11): 2506-2514.e4

9 Perzborn E, Strassburger J, Wilmen A, et al. In vitro and in vivo studies of the novel antithrombotic agent BAY 59-7939-an oral, direct Factor Xa inhibitor. J Thromb Haemost 2005;3(03):514-521

10 Luettgen JM, Knabb RM, He K, Pinto DJ, Rendina AR. Apixaban inhibition of factor Xa: Microscopic rate constants and inhibition mechanism in purified protein systems and in human plasma. J Enzyme Inhib Med Chem 2011;26(04):514-526
11 Mann KG, Nesheim ME, Church WR, Haley P, Krishnaswamy S. Surface-dependent reactions of the vitamin K-dependent enzyme complexes. Blood 1990;76(01):1-16

12 Baglin T. The role of the laboratory in treatment with new oral anticoagulants. J Thromb Haemost 2013;11(Suppl 1):122-128

13 Barrett YC, Wang Z, Frost C, Shenker A. Clinical laboratory measurement of direct factor Xa inhibitors: anti-Xa assay is preferable to prothrombin time assay. Thromb Haemost 2010; 104(06):1263-1271

14 Garcia D, Barrett YC, Ramacciotti E, Weitz JI. Laboratory assessment of the anticoagulant effects of the next generation of oral anticoagulants. J Thromb Haemost 2013;11(02):245-252

15 Hillarp A, Gustafsson KM, Faxälv L, et al. Effects of the oral, direct factor Xa inhibitor apixaban on routine coagulation assays and anti-FXa assays. J Thromb Haemost 2014;12(09):1545-1553

16 Dale BJ, Ginsberg JS, Johnston M, Hirsh J, Weitz JI, Eikelboom JW. Comparison of the effects of apixaban and rivaroxaban on prothrombin and activated partial thromboplastin times using various reagents. J Thromb Haemost 2014;12(11):1810-1815

17 Samuelson BT, Cuker A, Siegal DM, Crowther M, Garcia DA. Laboratory assessment of the anticoagulant activity of direct oral anticoagulants: a systematic review. Chest 2017;151(01): $127-138$

18 Gosselin R, Grant RP, Adcock DM. Comparison of the effect of the anti-Xa direct oral anticoagulants apixaban, edoxaban, and rivaroxaban on coagulation assays. Int J Lab Hematol 2016;38(05): 505-513

19 Jourdi G, Siguret V, Martin AC, et al. Association rate constants rationalise the pharmacodynamics of apixaban and rivaroxaban. Thromb Haemost 2015;114(01):78-86

20 Wong PC, White A, Luettgen J. Inhibitory effect of apixaban compared with rivaroxaban and dabigatran on thrombin generation assay. Hosp Pract (1995) 2013;41(01):19-25

21 Kim PY, Nesheim ME. Further evidence for two functional forms of prothrombinase each specific for either of the two prothrombin activation cleavages. J Biol Chem 2007;282(45):32568-32581

22 Kretz CA, Stafford AR, Fredenburgh JC, Weitz JI. HD1, a thrombindirected aptamer, binds exosite 1 on prothrombin with high affinity and inhibits its activation by prothrombinase. J Biol Chem 2006;281(49):37477-37485

23 Barenholz Y, Gibbes D, Litman BJ, Goll J, Thompson TE, Carlson RD. A simple method for the preparation of homogeneous phospholipid vesicles. Biochemistry 1977;16(12):2806-2810

24 Yau JW, Stafford AR, Liao P, Fredenburgh JC, Roberts R, Weitz JI. Mechanism of catheter thrombosis: comparison of the antithrombotic activities of fondaparinux, enoxaparin, and heparin in vitro and in vivo. Blood 2011;118(25):6667-6674

25 Mueck W, Lensing AW, Agnelli G, Decousus H, Prandoni P, Misselwitz F. Rivaroxaban: population pharmacokinetic analyses in patients treated for acute deep-vein thrombosis and exposure simulations in patients with atrial fibrillation treated for stroke prevention. Clin Pharmacokinet 2011;50(10):675-686

26 Frost C, Nepal S, Wang J, et al. Safety, pharmacokinetics and pharmacodynamics of multiple oral doses of apixaban, a factor Xa inhibitor, in healthy subjects. Br J Clin Pharmacol 2013;76(05): 776-786

27 Funatsu T, Iwatsuki Y, Kaku S. Darexaban has high sensitivity in the prothrombin time clotting test. J Thromb Haemost 2012;10 (04):703-705

28 Hemker HC, Al Dieri R, De Smedt E, Béguin S. Thrombin generation, a function test of the haemostatic-thrombotic system. Thromb Haemost 2006;96(05):553-561

29 Nesheim ME, Prendergast FG, Mann KG. Interactions of a fluorescent active-site-directed inhibitor of thrombin: dansylarginine N-(3-ethyl-1,5-pentanediyl)amide. Biochemistry 1979;18(06): 996-1003

30 Heldebrant CM, Butkowski RJ, Bajaj SP, Mann KG. The activation of prothrombin. II. Partial reactions, physical and chemical 
characterization of the intermediates of activation. J Biol Chem 1973;248(20):7149-7163

31 Bianchini EP, Orcutt SJ, Panizzi P, Bock PE, Krishnaswamy S. Ratcheting of the substrate from the zymogen to proteinase conformations directs the sequential cleavage of prothrombin by prothrombinase. Proc Natl Acad Sci U S A 2005;102(29): 10099-10104

32 Hibbard LS, Nesheim ME, Mann KG. Progressive development of a thrombin inhibitor binding site. Biochemistry 1982;21(10): 2285-2292

33 Krishnaswamy S, Mann KG, Nesheim ME. The prothrombinasecatalyzed activation of prothrombin proceeds through the intermediate meizothrombin in an ordered, sequential reaction. J Biol Chem 1986;261(19):8977-8984

34 Brufatto N, Nesheim ME. Analysis of the kinetics of prothrombin activation and evidence that two equilibrating forms of prothrombinase are involved in the process. J Biol Chem 2003;278 (09):6755-6764

35 Mueck W, Schwers S, Stampfuss J. Rivaroxaban and other novel oral anticoagulants: pharmacokinetics in healthy subjects, specific patient populations and relevance of coagulation monitoring. Thromb J 2013;11(01):10

36 Betz A, Krishnaswamy S. Regions remote from the site of cleavage determine macromolecular substrate recognition by the prothrombinase complex. J Biol Chem 1998;273(17):10709-10718

37 Nelder JA, Mead R. A Simplex method for function minimization. Comput J 1965;7:308-313

38 Sinha U, Lin PH, Edwards ST, et al. Inhibition of purified factor Xa amidolytic activity may not be predictive of inhibition of in vivo thrombosis: implications for identification of therapeutically active inhibitors. Arterioscler Thromb Vasc Biol 2003;23(06): 1098-1104

39 Salonen LM, Holland MC, Kaib PS, et al. Molecular recognition at the active site of factor Xa: cation- $\pi$ interactions, stacking on planar peptide surfaces, and replacement of structural water. Chemistry 2012;18(01):213-222

40 Roehrig S, Straub A, Pohlmann J, et al. Discovery of the novel antithrombotic agent 5-chloro-N-((5S)-2-oxo-3- [4-(3-oxomorpholin-4-yl)phenyl]-1,3-oxazolidin-5-ylmethyl)thiophene- 2carboxamide (BAY 59-7939): an oral, direct factor Xa inhibitor. J Med Chem 2005;48(19):5900-5908

41 Pinto DJ, Orwat MJ, Koch S, et al. Discovery of 1-(4-methoxyphenyl)-7-oxo-6-(4-(2-oxopiperidin-1-yl)phenyl)-4,5,6,7-tetrahydro-1H-pyrazolo[3,4-c]pyridine-3-carboxamide (apixaban, BMS562247), a highly potent, selective, efficacious, and orally bioavailable inhibitor of blood coagulation factor Xa. J Med Chem 2007;50(22):5339-5356

42 Steinberg BA, Becker RC. Structure-function relationships of factor Xa inhibitors: implications for the practicing clinician. J Thromb Thrombolysis 2014;37(02):234-241

43 Bhunia SS, Roy KK, Saxena AK. Profiling the structural determinants for the selectivity of representative factor-Xa and thrombin inhibitors using combined ligand-based and structure-based approaches. J Chem Inf Model 2011;51(08):1966-1985

44 Harenberg J, Krämer R, Giese C, Marx S, Weiss C, Wehling M. Determination of rivaroxaban by different factor Xa specific chromogenic substrate assays: reduction of interassay variability. J Thromb Thrombolysis 2011;32(03):267-271 